\title{
Creencias docentes de la enseñanza en la Universidad Autónoma de Aguascalientes (UAA)
}

\section{Teaching beliefs of the professors in the Autonomous University of Aguascalientes}

Silvia del Carmen Miramontes Zapata

Universidad Autónoma de Zacatecas (UAZ)

Pedro Palacios Salas

Universidad Autónoma de Aguascalientes (UAA)

\author{
Enrique Navarrete Sánchez \\ Universidad Autónoma del \\ Estado de México (UAEMéx)
}

\section{Resumen}

Las creencias docentes son uno de los factores principales para entender la práctica educativa de los profesores; la forma cómo enseña el docente se relaciona estrechamente con las representaciones que tiene sobre el contenido que enseña, el currículum, la organización escolar y los procesos de aprendizaje de los alumnos, entre otras cosas. Para poder analizar la práctica educativa de los docentes de la Universidad Autónoma de Aguascalientes (UAA), se realizó esta investigación cuyos objetivos fueron: 1. Describir las características propias de profesionalización y formación de los docentes, tales como años de experiencia, tiempo dedicado a esta actividad, grado académico, área de especialización, asignaturas impartidas y función principal dentro de la institución. 2. Conocer las perspectivas de enseñanza dominante de los profesores de la UAA, sin separar sus tres componentes: creencias, propósitos y acciones. 3. Comparar los distintos tipos de perspectivas dominantes de los profesores con algunas características propias de la profesionalización y formación docente. La muestra estuvo conformada por 73 docentes de ambos sexos que forman parte de la planta docente de la universidad; se aplicó el Inventario de Perspectivas de Enseñanza (IPE). Los resultados obtenidos indican que la perspectiva dominante de los profesores es la de transmisión, es decir, para los profesores de la UAA un buen profesor es aquel que domina el contenido de la materia, tiene objetivos claros, presenta en forma oportuna retroalimentación a sus estudiantes y tiene un fuerte compromiso con los contenidos.

Palabras clave: creencias, docentes, práctica, educativa, universidad.

Nota del autor

Silvia del Carmen Miramontes Zapata, Unidad Académica de Psicología, Universidad Autónoma de Zacatecas (UAZ). Pedro Palacios Salas, Departamento de Psicología, Universidad Autónoma de Aguascalientes (UAA). Enrique Navarrete Sánchez, Facultad de Ciencias de la Conducta, Universidad Autónoma del Estado de México (UAEMéx).

La correspondencia en relación con este artículo debe dirigirse a Silvia del Carmen Miramontes Zapata, Universidad Autónoma de Zacatecas, Jardín Juárez \#147, Centro Histórico C.P. 98000 , Zacatecas, Zacatecas, México.

Dirección electrónica: silvia.miramontes@uaz.edu.mx 


\begin{abstract}
Teacher's beliefs are the main factor to understand the educational practice. The teaching style is closely related to the representations that a teacher has about the teaching contents, the curriculum, the school organization and the learning process of the students. Among other things, in order to analyze the educational practice of teachers from Autonomous University of Aguascalientes (UAA), the current research was carried out, its objectives were: 1. To describe the professionalization and teachers' training characteristics such as years of experience, time devoted to this activity, academic degree, specialty area, subjects that are taught as well as his/ her main function in the institution. 2. To know the predominant educational perspectives of teachers from UAA without separating the perspectives components (beliefs, purposes and actions). 3. To compare the different predominant perspectives with some characteristics which are typical aspects of professionalization and teachers training. The sample consisted of 73 teachers of both sexes that are part of the staff; the Teaching Perspectives Inventory was administered. Some of the findings suggest that the perspective of transmission is the predominant one, this means that for UAA teachers, a good teacher is who masters the subject contents, has clear objectives, presents a convenient feedback to his/ her students and has a strong commitment with the contents.
\end{abstract}

Keywords: beliefs, teachers, practice, educational, university.

La investigación educativa se ha transformado en las últimas décadas. Anteriormente, al efectuar este tipo de investigación, en principio, se realizaba con relación a los alumnos y sus procesos desde una perspectiva cuantitativa; en años recientes se diversifica y ahora se investiga a los docentes desde diferentes perspectivas metodológicas, tanto cuantitativas como cualitativas, su práctica educativa y su rol como docente. Hoy por hoy se habla de la evaluación docente y su rol en el llamado nuevo modelo educativo. De esta manera, resulta por demás importante para entender este rol, conocer sus creencias como docentes sobre el proceso de enseñanza para entender su práctica educativa.

Los modelos educativos actuales han modificado el papel del docente, es así que se transita de un modelo llamado "tradicional", cuya característica es que el centro del proceso de enseñanza y aprendizaje lo tiene el docente como transmisor de la información, a los modelos actuales, donde se concibe a un profesor reflexivo y mediador del proceso de aprendizaje de sus alumnos. Por tal razón, es de suma relevancia poder establecer cuáles son las creencias que tiene el docente sobre la enseñanza, ya que como lo mencionan Clark y Peterson, (1990, p. 445), “en gran medida, lo que los docentes hacen es consecuencia de lo que piensan. Dados los cambios producto de la modificación del modelo educativo, la forma de enseñar se modificó".

De esta manera, la forma en la que el docente concibe la enseñanza es en la que la planea y la ejecuta en el salón de clases; de acuerdo a Trigwell y Prosser (1991), las creencias que 
los profesores tienen sobre la enseñanza, el aprendizaje y su práctica docente produce también una estrecha relación con el aprendizaje de los alumnos. Es decir, el desempeño del estudiante se ve influenciado por las creencias del profesor. Así, las creencias constituyen verdades personales, derivadas de la experiencia o de la fantasía, con un fuerte componente afectivo y evaluativo. Se manifiestan en las declaraciones verbales, escritas o en las acciones y, por lo tanto, condicionan los procesos de decisión (Pajares, 1992). Igualmente, la creación de una creencia y todo lo que ella conlleva se encuentra determinada en principio por la cultura general que rodea al sujeto y que, en cierta manera, va a determinar esa generación de pensamientos, actitudes, aptitudes y opiniones (Díaz, Jansson \& Neira, 2012). Para Martínez (2013), las creencias se constituyen en verdades idiosincráticas que no requieren una condición de verdad contrastada, dado que representan datos, supuestos y opiniones propias o transmitidas por otros y surgidas desde los saberes del sentido común.

Las creencias docentes abarcan las posturas o posicionamientos que los profesores tienen respecto a su práctica educativa (Ponte, 1999, citado en Solís, 2015). Para Kagan (1992), las creencias y concepciones ayudan a los profesores a controlar la incertidumbre y la ambigüedad que pueda darse en su práctica. Así, los maestros construyen creencias en función de tres factores: (1) sobre los estudiantes, tomando en cuenta las características del grupo y el contexto en el que se encuentran; (2) sobre los aspectos fundamentales que deben enseñar, el contenido y la disciplina a la cual corresponden; y (3) sobre sus experiencias previas como estudiantes (Ferreyra, 2012). No obstante, puede surgir un problema cuando los docentes se aproximan a la evaluación con dichas creencias, porque las creencias que los profesores tienen sobre sus alumnos no siempre están en correspondencia con el entorno donde los estudiantes aprenden (López \& Basto, 2010).

Los objetivos que guiaron la presente investigación fueron los que a continuación se enumeran:

1. Describir las características propias de profesionalización y formación de los docentes, tales como años de experiencia, tiempo dedicado a esta actividad, grado académico, área de especialización, asignaturas impartidas y función principal dentro de la institución.

2. Conocer las perspectivas de enseñanza dominante de los profesores de la UAA, sin separar sus tres componentes (creencias, propósitos y acciones).

3. Comparar los distintos tipos de perspectivas dominantes de los profesores con algunas características propias de la profesionalización y formación docente.

\section{Método}

Para alcanzar los objetivos previamente señalados, se realizó una investigación descriptiva, transversal, con un diseño no experimental. 


\section{Muestra}

La población a partir de la cual se trabajó fue la planta docente de la UAA. La oferta educativa de la UAA está constituida por 65 programas académicos de pregrado, de los cuales 14 son ingenierías y 51 licenciaturas; además, ofrece 14 especialidades médicas en coordinación con instituciones de salud en el estado; una especialidad a distancia, 16 maestrías y 10 doctorados. En la UAA, a diferencia de otras universidades, está organizada por áreas y no por facultades, como la mayoría de las universidades (UAA, 2017).
La muestra estuvo conformada por 73 docentes de ambos sexos que forman parte de la planta docente de la universidad, quienes imparten principalmente en las áreas de psicología (18 participantes), artes (16 participantes), salud (12 participantes), investigación (9 participantes), diseño/ arquitectura (8 participantes), ingeniería (3 participantes), administración (2 participantes), química (1 participante) y en lenguas (1 participante). Dos participantes del estudio no respondieron a este rubro). En la tabla 1, puede encontrarse la información correspondiente a la frecuencia y porcentaje de este rubro. El tipo de muestreo utilizado fue no probabilístico voluntario.

Tabla 1

Áreas en las que principalmente enseñan los docentes participantes

\begin{tabular}{lccc}
\hline & Frecuencia & Porcentaje & Porcentaje válido \\
\hline Psicología & 18 & 24.7 & 25.4 \\
Investigación & 9 & 12.3 & 12.7 \\
Sociales & 1 & 1.4 & 1.4 \\
Ingeniería & 3 & 4.1 & 4.2 \\
Administración & 2 & 2.7 & 2.8 \\
Química & 1 & 1.4 & 1.4 \\
Salud & 12 & 16.4 & 16.9 \\
Diseño/arquitectura & 8 & 11.0 & 11.3 \\
Artes & 16 & 21.9 & 22.5 \\
Lenguas & 1 & 1.4 & 1.4 \\
Casos perdidos & 2 & 2.7 & \\
Total & & & 100.0 \\
\hline
\end{tabular}


Con la intención de formar grupos con un número de profesores relativamente homogéneo con respecto a la cantidad de profesores en cada grupo, se reagruparon en áreas afines, quedando conformados de la siguiente forma: Psicología (18 participantes), Investigación y sociales (10 participantes), Ingeniería, diseño, arquitectura y administración (13 participantes) y, por último, Salud y química (13 participantes), ello para análisis posteriores.

\section{Instrumento}

Para alcanzar los objetivos previamente señalados, se aplicó el Inventario de Perspectivas de Enseñanza (IPE), (Pratt \& Collins, 2001). Este inventario fue desarrollado por los autores con el fin de determinar perfiles de los profesores con respecto a identificar qué creen, que se proponen lograr y que hacen los profesores cuando enseñan. Originalmente, fue elaborado en inglés con el nombre ya antes mencionado, así como su versión en español con el nombre Inventario de Perspectivas de Enseñanza (IPE). Dicho inventario está conformado por 45 reactivos con una categoría de respuestas que va de 1 al 5, subdividido en cinco perspectivas: Transmisión, Aprendizaje, Desarrollo, Acompañamiento y Reforma social, con nueve reactivos cada una. La tabla 2 muestra la descripción de cada tipo de perspectivas. Cada perspectiva cuenta con nueve reactivos o ítems del instrumento.

Tabla 2

Descripción de las perspectivas de enseñanza (extraído de Organización del Bachillerato Internacional, 2013)

\begin{tabular}{ll}
\hline Perspectiva & \multicolumn{1}{c}{ Descripción } \\
\hline Transmisión & $\begin{array}{l}\text { Los docentes con esta perspectiva tienen un fuerte compromiso con el contenido de } \\
\text { la asignatura; la principal responsabilidad del docente es presentar el contenido a los } \\
\text { alumnos de manera precisa y eficaz. }\end{array}$ \\
Aprendizaje & $\begin{array}{l}\text { La enseñanza eficaz es un proceso de enculturación de los alumnos en un sistema de } \\
\text { normas sociales y formas de trabajo. }\end{array}$ \\
Desarrollo & $\begin{array}{l}\text { El objetivo principal es ayudar a los alumnos a desarrollar estructuras cognitivas cada } \\
\text { vez más complejas y sofisticadas para comprender el contenido. }\end{array}$ \\
Acompañamiento & $\begin{array}{l}\text { Los alumnos se educan al saber que: (a) pueden obtener buenos resultados en el } \\
\text { aprendizaje si se esfuerzan, y (b) su logro es producto de su propio esfuerzo y ca- } \\
\text { pacidad. }\end{array}$ \\
& $\begin{array}{l}\text { La enseñanza eficaz busca cambiar la sociedad de manera sustancial. Desde este } \\
\text { punto de vista, el objeto de la enseñanza es colectivo más que individual. Los buenos } \\
\text { docentes hacen que los alumnos tomen conciencia de los valores y las ideologías que } \\
\text { están presentes en los textos y las prácticas comunes dentro de su disciplina. }\end{array}$ \\
\hline
\end{tabular}


A su vez, cada perspectiva puede ser observada desde tres dimensiones, que representan las Creencias, las Intenciones y las Acciones que los profesores realizan cuando enseñan. Cada una de las dimensiones se responde de acuerdo con una escala de cinco puntos.

Las Creencias, con una categoría de respuesta que va de 1 (Completamente en desacuerdo) a 5 (Completamente de acuerdo). Mientras que las dimensiones Propósitos y Acciones emplean una categoría de cinco puntos que va de 1 (Nunca) a 5 (Siempre).

Los profesores responden al instrumento marcando con una equis, " $X$ ", en el cuadro que indica su respuesta a cada una de las afirmaciones del inventario. La puntuación para cada una de las perspectivas se obtiene al sumar las equis marcadas para cada uno de los reactivos. La confiabilidad de la prueba, al menos en su versión en inglés - calculada a partir de la prueba alpha de Cronbach, y para una muestra de más de 60,000 profesores que respondieron la versión online del TPI -, fluctúa de .70 para la perspectiva de Desarrollo a .83 para la de Reforma Social, por lo cual puede decirse que es aceptable (Nunnally, \& Bernstein, 1994). Asimismo, las correlaciones interescalas van de .15 entre las subescalas Transmisión y Acompañamiento a .58 entre las subescalas Aprendizaje y Desarrollo.

El TPI ha sido respondido por profesores de diferentes especialidades, de educación superior, educadores de adultos, dietólogos, estudiantes de licenciatura para formar profesores, profesores de escuelas, así como estudiantes en formación para la enseñanza de idiomas.

Con respecto a su validez, ésta ha sido explorada mediante la validez de facie, realizada mediante el juicio de 75 profesores expertos, con una concordancia de 95\%. Se sugiere que los reactivos son representaciones razonables de las perspectivas que pretenden contener. La validez explorada mediante el análisis factorial mostró que el IPE está constituido por los cinco factores o dimensiones propuestas, así como que los reactivos quedaron asignados adecuadamente a los factores que conforman el inventario.

Para este estudio, se utilizó el TPI que fue traducido al español y retraducido al inglés por cinco profesores bilingües, con el fin de observar si el instrumento sería equivalente a su versión en inglés. Enseguida, los reactivos fueron sometidos a una evaluación por parte de diez profesores expertos: cinco de la Facultad de Educación y cinco de la Facultad de Psicología, actualmente en servicio en la Universidad Autónoma de Yucatán (UADY). Los resultados mostraron una concordancia superior a $85 \%$, por lo cual se consideró adecuado para realizar la investigación (Canto, 2010).

\section{Procedimiento}

Para la aplicación de los instrumentos, se entregó a cada profesor un ejemplar impreso de los mismos, se les explicaron los objetivos que perseguía el estudio, se reiteró el carácter confidencial de los resultados y se les pidió que 
lo respondieran lo más honestamente posible. La aplicación de los mismos fue aproximadamente de 15 min y fueron aplicados en áreas designadas por la universidad para dicho fin.

\section{Resultados}

Formación y profesionalización de la práctica

\section{docente de los participantes}

Los datos fueron analizados a través del programa estadístico SPSS, Versión 12. Debido a que el tipo de muestreo fue voluntario, las características no eran conocidas con antelación por los participantes. Debido a esto, en primer lugar, se expondrán los resultados relativos a la formación y profesionalización de los participantes en términos de frecuencias $y$ porcentajes.

Con relación a la función o papel principal dentro de la universidad, 63 participantes $(86.3 \%)$, señalan como su función principal la docencia, seguida por la investigación (seis participantes, 8.2\%) y la gestión (cuatro participantes, $5.5 \%$ ).

Con respecto a las horas dedicadas a la docencia a la semana, 26 profesores (equivalente a $35.6 \%$ ), dedican entre 11 y $20 \mathrm{~h}$ a esta actividad; $23(31 \%)$ de 1 a 10 h; 17 (23\%) le dedica entre 21 a $30 \mathrm{~h}$; mientras que el resto, es decir, siete docentes $(9.6 \%)$ le dedican entre 31 y $40 \mathrm{~h}$. Cabe señalar que cuando los profesores mencionaron dichos tiempos, no especificaron si era con relación a las horas que pasaban dando clases o incluían también las horas de preparación de las mismas, como ocurre en algunas universidades.
Acerca del grado académico más alto obtenido, la mayoría de los profesores cuenta con un grado de maestría (38 docentes, equivalente a $52.1 \%$ ), en segundo lugar, quince docentes cuentan con el grado de licenciatura (20\%), catorce con el grado de doctor (19.2\%), y sólo $6(8.2 \%)$, cuentan con especialidad. Estos resultados son similares a otros encontrados en estudios realizados en otras universidades, (véase, por ejemplo, Hernández, 2015), en donde se encontraba que el grado máximo de formación de la mayoría de los profesores era de maestría.

Con relación a la experiencia docente de los participantes, medida en el número de años dedicados a esta actividad, 20 participantes $(30 \%)$, señalan que entre uno y ocho años, que es la misma cantidad de profesores que llevan entre nueve y 16 años, 18 profesores (25\%) han dedicado 25 años o más a esta actividad, y 11 profesores (15\%) entre 17 y 24 años.

Todos los resúmenes mencionados, pueden encontrarse en la tabla 3.

Sobre el área de especialización de acuerdo con el último grado académico, la mayor parte de los docentes se concentra en el área de psicología, artes (15 docentes en cada área, equivalente a $21.1 \%$, respectivamente) y salud (10 docentes, equivalente a 14.1\%). El resto se encuentra distribuido en áreas diversas, mismas que pueden verse en la tabla 4. 
Tabla 3

Características de los participantes

\begin{tabular}{lccccccc}
\hline \multicolumn{2}{c}{$F D U$} & \multicolumn{2}{c}{$H D D S$} & \multicolumn{2}{c}{$G A O$} & \multicolumn{2}{c}{$E D$} \\
\hline Función & Frecuencia & $\begin{array}{c}\text { Número } \\
\text { horas }\end{array}$ & Frecuencia & $\begin{array}{c}\text { Grado } \\
\text { académico }\end{array}$ & Frecuencia & Años & Frecuencia \\
Docencia & $63(86.3 \%)$ & $\begin{array}{c}\text { de } 1 \mathrm{a} \\
10, \text { horas }\end{array}$ & $23(31 \%)$ & Licenciatura & $15(20 \%)$, & 1 a 8 & $22(30 \%)$ \\
Investigación & $6(8.2 \%)$ & $\begin{array}{c}11 \text { y } 20, \\
\text { horas }\end{array}$ & $26(35.6 \%)$ & Especialidad & $6(8.2)$ & 9 a 16 & $22(30 \%)$ \\
Gestión & $4(5.5 \%)$. & $\begin{array}{c}21 \text { a } 30, \\
\text { horas } \\
31 \text { y } 40, \\
\text { horas }\end{array}$ & $7(23 \%)$ & Maestría & $38(52.1 \%)$ & 17 a 24 & $11(15 \%)$ \\
\hline
\end{tabular}

Nota: FDU= Función o papel principal dentro de la universidad; HDDS= Horas dedicadas a la docencia a la semana; GAO= Grado académico más alto obtenido; ED= Experiencia docente.

Tabla 4

Área de especialización de acuerdo al último grado de estudios más alto

\begin{tabular}{lc}
\hline Área & Frecuencia (\% válido) \\
\hline Salud & $10(14.1 \%)$ \\
Ingeniería & $3(4.2 \%)$ \\
Sociales & $6(8.5 \%)$ \\
Lenguas & $2(2.8 \%)$ \\
Psicología & $15(21.1 \%)$ \\
Administración & $3(4.2 \%)$ \\
Educación/pedagogía & $9(12.7 \%)$ \\
Artes & $15(21.1 \%)$ \\
Química & $1(1.4 \%)$ \\
Diseño/arquitectura & $7(9.9 \%)$ \\
No contesta & 2 \\
Total & $73(100 \%)$ \\
\hline
\end{tabular}

\section{Tipos de perspectivas de enseñanza} dominantes

A continuación se presentarán los resultados relativos a los tipos de perspectivas dominantes de los docentes de la UAA. Para poder conocer los tipos de perspectivas, se realizó un análisis descriptivo de todos los valores que correspondían a cada una de las perspectivas en las tres dimensiones consideradas dentro del instrumento (Creencias, Propósitos y Acciones). Dichos resultados se presentan en la tabla 5. 
Cabe hacer notar que los promedios en todas las perspectivas son muy similares, por lo tanto, como lo mencionaron Pratt, Colling y Selinger (2001), no existe en los profesores una perspectiva única, por lo cual es necesario hablar de una dominante. Como puede notarse en la tabla 5, la perspectiva dominante de los profesores es la de Transmisión, es decir, para los profesores de la UAA un buen profesor es aquel que domina el contenido de la materia, tiene objetivos claros, presenta en forma oportuna retroalimentación a sus estudiantes y tiene un fuerte compromiso con los contenidos. La segunda perspectiva dominante es la de Aprendizaje: los profesores son expertos en lo que enseñan, y eso lo demuestran tanto en el aula como en los otros lugares donde desempeñan su profesión. Su principal objetivo es conseguir que los estudiantes desarrollen su máximo potencial en forma independiente. La perspectiva menos dominante es la de Reforma social.

Tabla 5

Estadísticos descriptivos de los tipos de perspectiva de aprendizaje

\begin{tabular}{lccc}
\hline Tipo de perspectiva & Valores mínimos & Valores máximos & Media $(D . E)$. \\
\hline Transmisión & 29.00 & 45 & $37.2329(4.25)$ \\
Aprendizaje & 28.00 & 45 & $36.9863(4.31)$ \\
Desarrollo & 25.00 & 45 & $35.1806(4.47)$ \\
Acompañamiento & 19.00 & 44 & $36.6712(5.2)$ \\
Reforma social & 14 & 44 & $33(6.2)$ \\
\hline
\end{tabular}

\section{Diferencias en relación con los tipos de} perspectivas dominantes y características de la formación y profesionalización docentes

Para saber si había diferencias significativas en relación con algunas de las características relativas a la formación y profesionalización de los docentes, se realizó un análisis utilizando la prueba de Kruskal-Wallis.
Los resultados pueden verse en la tabla 6 . Con relación al tipo de perspectiva y el área de especialización, no se encontraron diferencias significativas, tampoco con la función principal de los profesores, ni con el grado académico más alto obtenido, ni con los años de experiencia docente. 
Tabla 6

Relación tipo de perspectiva y área de especialización

\begin{tabular}{|c|c|c|c|c|c|c|}
\hline $\begin{array}{l}\text { Área de } \\
\text { profesionalización }\end{array}$ & & Transmisión & Aprendizaje & Desarrollo & Acompañamiento & $\begin{array}{c}\text { Reforma } \\
\text { social }\end{array}$ \\
\hline $\begin{array}{l}\text { Área de } \\
\text { especialización }\end{array}$ & $\begin{array}{c}X^{2} \\
G I \\
\text { Significancia }\end{array}$ & $\begin{array}{c}.314 \\
2 \\
.854\end{array}$ & $\begin{array}{c}.580 \\
2 \\
.748\end{array}$ & $\begin{array}{l}1.878 \\
2 \\
.391\end{array}$ & $\begin{array}{l}.049 \\
2 \\
.976\end{array}$ & $\begin{array}{c}.525 \\
2 \\
.769\end{array}$ \\
\hline Función principal & $\begin{array}{c}X^{2} \\
G l \\
\text { Significancia }\end{array}$ & $\begin{array}{c}.880 \\
3 \\
.830\end{array}$ & $\begin{array}{c}.520 \\
3 \\
.914\end{array}$ & $\begin{array}{l}.327 \\
3 \\
.955\end{array}$ & $\begin{array}{c}1.436 \\
3 \\
.697\end{array}$ & $\begin{array}{c}.711 \\
3 \\
.871\end{array}$ \\
\hline Grado académico & $\begin{array}{c}X^{2} \\
G l \\
\text { Significancia }\end{array}$ & $\begin{array}{c}.695 \\
3 \\
.874\end{array}$ & $\begin{array}{c}1.206 \\
3 \\
.751\end{array}$ & $\begin{array}{c}3.659 \\
3 \\
.301\end{array}$ & $\begin{array}{l}6.393 \\
3 \\
.094\end{array}$ & $\begin{array}{c}.897 \\
3 \\
.826\end{array}$ \\
\hline $\begin{array}{l}\text { Años de } \\
\text { experiencia }\end{array}$ & $\begin{array}{c}X^{2} \\
G / \\
\text { Significancia }\end{array}$ & $\begin{array}{l}.436 \\
3 \\
.933\end{array}$ & $\begin{array}{l}1.726 \\
3 \\
.631\end{array}$ & $\begin{array}{l}4.173 \\
3 \\
.243\end{array}$ & $\begin{array}{l}2.617 \\
3 \\
.455\end{array}$ & \\
\hline
\end{tabular}

$P<0.05$

\section{Conclusiones}

Al realizar la presente investigación se concluye, con base en los objetivos, que:

1. El $52.1 \%$ de docentes cuenta con grado de Maestría, de $86.3 \%$, su función principal es la docencia, $35.6 \%$ dedica entre 11 y 20 h a esta actividad y $30 \%$, señala que entre uno y ocho años tienen dedicados a la docencia.

2. La perspectiva dominante de los profesores es la de Transmisión, es decir, para los profesores de la UAA un buen profesor es aquel que domina el contenido de la materia, tiene objetivos claros, presenta en forma oportuna retroalimentación a sus estudiantes y tiene un fuerte compromiso con los contenidos.

3. No se encontraron diferencias significativas, tampoco con la función principal de los profesores ni con el grado académico más alto obtenido, tampoco con los años de experiencia docente.

Finalmente, se puede decir que el docente universitario, en particular el de la UAA, tienen claro su papel como docente en la formación de profesionistas.

\section{Referencias}

Clark, C., \& Peterson, P. (1990). Procesos de pensamiento de los docentes. En M. Wittrock (Ed.), La investigación de la enseñanza, Vol. 3. Barcelona: Paidós.

Díaz, C., Jansson, L., \& Neira, A. (2012). Percepciones de profesores y estudiantes chilenos de educación media acerca del papel de la tecnología en la clase de inglés como lengua extranjera. Revista Lasallista de Investigación, 8(2), 3-60. 
Recuperado de http://www.redalyc.org/ pdf/695/69522607006.pdf

Ferreyra, A. (2012). Creencias y concepciones docentes sobre la evaluación de los aprendizajes en el contexto universitario (Tesis de Licenciatura). Pontificia Universidad Católica del Perú, Lima, Perú: Recuperado de http://tesis.pucp.edu.pe/repositorio/bitstream/handle/123456789/4425/ FERREYRA_DIAZ_ANA_CREENCIAS. pdf? sequence $=1$ \&isAllowed $=\mathrm{y}$

Kagan, D. (1992). Implications of Research on Teacher Belief. Educational Psychologist, 27(1), 65-90. https://doi.org/10.1207/ s15326985ep2701_6

López, B., \& Basto, S. (2010). Desde las teorías implícitas a la docencia como practica reflexiva. Educación y Educadores, 13(2), 275-291. Recuperado de http://www.scielo. org.co/pdf/eded/v13n2/v13n2a07.pdf

Martínez, N. (2013). Las creencias de los profesores universitarios sobre evaluación del aprendizaje. Diálogos, 7(12), 45-66. Recuperado de http:/www.redicces.org. sv/jspui/bitstream/10972/2096/2/Las\%20 creencias $\% 20 \mathrm{de} \% 2010 \mathrm{~s} \% 20$ profesores $\% 20$ universitarios $\% 20$ sobre $\% 20$ evaluacion $\% 20$ del\%20aprendizaje.pdf

Nunnally, J. C., \& Bernstein, I. H. (1994). Psychometric theory (3rd ed.). New York: McGraw-Hill.

Organización del Bachillerato Internacional. (2013). Resumen de la investigación El docente profesional del IB: identificación, análisis y caracterización de sus atributos pedagógicos, perspectivas y principios.
Recuperado de http://www.ibo.org/ globalassets/publications/ibesearch/ continuum/teacher_summary_s.pdf

Pajares, F. (1992). Teachers' Beliefs and Educational Research: Cleaning Up a Messy Construct. [Creencias de los profesores y la investigacion educativa: limpiando un constructo confuso]. Review of Educational Research, 62(3), 307-332. Recuperado de http://rer.sagepub.com/content/62/3/307.full. pdf + html.

Pratt, D., Collins, J., \& Selinger, S. (2001). Development and Use of The Teaching Perspectives Inventory (TPI). Trabajo inédito presentado en la conferencia anual de AERA de 2001 en Seattle, Washington, (EE. UU.). Recuperado de http://www.one45.com/ teachingperspectives/PDF/development1.pdf

Solís, C. (2015). Creencias sobre enseñanza y aprendizaje en docentes universitarios: Revisión de algunos estudios. Propósitos y Representaciones, 3(2), 227-260. doi: http:// dx.doi.org/10.20511/pyr2015.v3n2.83

Trigwell, K., \& Prosser, M. (1991). Improving the quality of student learning: the influence of learning context and student learning outcomes. Higher Education, 22, 251-266. Recuperado de https://link.springer.com/ article/10.1007\%2FBF0013229

Recibido: 06/07/2017

Revisado: $15 / 12 / 2017$

Aceptado: 04/05/2018 